\title{
Formulas for series computations
}

\section{Report}

\section{Author(s):}

Bronstein, Manuel

Publication date:

1991

Permanent link:

https://doi.org/10.3929/ethz-a-000609400

Rights / license:

In Copyright - Non-Commercial Use Permitted

Originally published in:

ETH, Eidgenössische Technische Hochschule Zürich, Departement Informatik, Institut für Wissenschaftliches Rechnen 169 
EH

Eidgenössische

Technische Hochschule

Zürich
Departement Informatik Institut für

Wissenschaftliches Rechnen
Manuel Bronstein

\section{Formulas for} Series Computations

Eidg. Techn. Hochschule Zürich Informatikbibliothek

\author{
Entzontum
}

$\mathrm{CH}$-86ag zürich

October 1991

\title{
169
}


Preprint to be published in Applicable Algebra in Engineering, Communication and Computing

Authors' address:

Institut für Wissenschaftliches Rechnen ETH Zentrum $\mathrm{CH}-8092$ Zurich, Switzerland. - e-mail: bronstein@inf.ethz.ch

(C) 1991 Departement Informatik, ETH Zürich 


\begin{abstract}
We describe an algorithm that computes polynomials whose roots are the coefficients of any specific order of the Laurent series of a rational function. The algorithm uses only rational operations in the coefficient field of the function. This allows us to compute with the principal parts of the Laurent series, in particular with the residues of the function, without factoring or splitting its denominator. As applications, we get a generalisation of the residue formulas used in symbolic integration algorithms to $n^{\text {th }}$-order formulas. We also get an algorithm that computes explicitly the principal parts at all the poles simultaneously, while computing in the field generated by the coefficients of the series instead of the one generated by the poles of the function. This yields an improved version of the necessary conditions for the various cases of Kovacic's algorithm, that expresses those conditions in the smallest possible extension field.
\end{abstract}

Keywords: Power series, Residues.

\title{
1 Introduction
}

The residues of complex functions at their isolated singularities arise from their Laurent series expansions at those singularities. They are used in complex integration and definite real integration [5]. They can also be defined algebraically for rational functions over an algebraically closed field as some coefficients of the $P$-adic expansions at the poles of the function [3]. These "algebraic" residues, which are equivalent to the analytic residues in the complex rational function case, are used in symbolic integration algorithms (e.g. [7]). Furthermore, some other coefficients of the $P$-adic expansions, which we call the "higher-order residues" are used in algorithms that look for closed form solutions of differential equations [1]. The main computational problem is that computing the Laurent series of a rational function over a field $K$ requires extensive computations in an algebraic closure of $K$, or more precisely in the splitting field of the denominator of the function, which is in general ineffective. This is particularly wasteful since it can happen that the field generated by the residues has a much lower degree over $K$ than this splitting field. For this reason, a special case formula has been devised $[6,7]$ which computes a polynomial whose roots are exactly the residues of a rational function, provided that it has only simple poles. In this paper, we 
generalize this formula and obtain a family of polynomials whose roots are precise subsets of the coefficients of the Laurent series of a rational function with poles of arbitrary order. Together, these polynomials cover all the coefficients corresponding to negative exponents in the series. All the computations are done in the coefficient field of the function and only involve rational operations. As applications we can determine the smallest extension field containing the residues of a function, and compute the principal parts of the Laurent series at all its poles while computing only in the field generated by the coefficients of the series. This field is always contained in the field generated by the poles of the function and can often be much smaller. At the end, we apply this algorithm to test necessary conditions for the 3 cases of Kovacic's algorithm [1] in the smallest possible extension field.

\section{Taylor and Laurent Series}

Let $k$ be an algebraically closed field of characteristic 0 and $x$ be an indeterminate over $k$. We recall in this section the definition of the $P$-adic series expansions, and show that they are the algebraic analogues of the Taylor and Laurent series for complex functions. Notation: The only derivation used in this paper is ' $=d / d x$, and $g^{(i)}$ denotes the $i^{\text {th }}$ derivative of $g$ with respect to $x$. We also write $g^{\prime}$ and $g^{\prime \prime}$ for $g^{(1)}$ and $g^{(2)}$ respectively.

Let $a \in k$. Recall that the a-valuation is the map $\nu_{a}: k(x) \backslash$ $\{0\} \rightarrow Z$ defined by.

(a) for $A \in k[x] \backslash\{0\}, \nu_{a}(A)$ is the unique integer $n$ such that $(x-a)^{n} \mid A$ and $(x-a)^{n+1}$ does not divide $A$;

(b) for $A, B \in k[x] \backslash\{0\}, \nu_{a}(A / B)=\nu_{a}(A)-\nu_{a}(B)$.

The local ring at $a$ is defined by

$$
\mathcal{O}_{a}=\left\{f \in k(x) \text { s.t. } \nu_{a}(f) \geq 0\right\} .
$$

Finally, the value at $a$ is the map $\phi_{a}: \mathcal{O}_{a} \rightarrow k$ defined by: let $f \in \mathcal{O}_{a}$ and write $f=A / B$ with $A, B \in k[x],(A, B)=(1)$ and $B \neq 0$. Since $f \in \mathcal{O}_{a},(B, x-a)=(1)$, so there exist $C, D \in k[x]$ such that $B C+(x-a) D=1$. Then,

$$
\phi_{a}(f)=A C \bmod (x-a) .
$$


It is easily checked that $\phi_{a}$ is well-defined on $\mathcal{O}_{a}$, and that $\phi_{a}(f)=$ $A(a) C(a)=A(a) / B(a)$. By analogy with complex functions, we write $f(a)$ for $\phi_{a}(f)$.

Let $f \in \mathcal{O}_{a}$, and define the sequence $S_{f}=\left(f_{n}\right)_{n \geq 0}$ by

$$
\left\{\begin{array}{l}
f_{0}=f \\
f_{n}=\left(f_{n-1}-f_{n-1}(a)\right) /(x-a) \text { for } n \geq 1 .
\end{array}\right.
$$

This definition corresponds to the construction described in [3] for the $P$-adic series. The following Lemma shows that $S_{f}$ is welldefined:

Lemma 1 Let $f \in \mathcal{O}_{a}$ and $S_{f}$ be given by (1). Then, $f_{n} \in \mathcal{O}_{a}$ for $n \geq 0$.

Proof. By induction on $n$. $f_{0}=f \in \mathcal{O}_{a}$, so let $n \geq 0$ and suppose that $f_{n} \in \mathcal{O}_{a}$. Then $\nu_{a}\left(f_{n}\right) \geq 0$, so $\nu_{a}(g) \geq 0$ where $g=f_{n}-f_{n}(a)$. But $g(a)=f_{n}(a)-f_{n}(a)=0$, so $\nu_{a}(g) \geq 1$, so $\nu_{a}(g /(x-a))=$ $\nu_{a}(g)-1 \geq 0$, so $f_{n+1}=g /(x-a) \in \mathcal{O}_{a}$.

Definition 1 Let $f \in \mathcal{O}_{a}$. The P-adic expansion of $f$ at $x=a$ is the series

$$
\sum_{n=0}^{\infty} a_{n}(x-a)^{n}=a_{0}+a_{1}(x-a)+a_{2}(x-a)^{2}+\cdots \quad \in k[[x]]
$$

where $a_{n}=f_{n}(a)$ and the $f_{i}$ 's are given by (1).

In order to show that these expansions are the usual Taylor series in the complex case, we prove that they satisfy Taylor's formula.

Lemma 2 Let $F$ be a field, $x$ an indeterminate over $F, a \in F$, $f \in F(x)$, and $g=(x-a) f$. Then,

$$
g^{(n)}=(x-a) f^{(n)}+n f^{(n-1)} \quad \text { for any integer } n>0 .
$$

Proof. $g=(x-a) f$, so

$$
g^{(n)}=\sum_{i=0}^{n}\left(\begin{array}{c}
n \\
i
\end{array}\right)(x-a)^{(i)} f^{(n-i)} .
$$

Since $(x-a)^{(i)}=0$ for $i \geq 2$, the only nonzero terms in the above sum are the terms for $i=0$ and $i=1$, and the Lemma follows. 
Lemma 3 (Taylor's formula) Let $f \in \mathcal{O}_{a}$ and $S_{f}$ be given by (1). Then,

$$
f_{n}(a)=\frac{f^{(n)}(a)}{n !} \text { for } n \geq 0
$$

Proof. $f_{0}(a)=f(a)=f^{(0)}(a)$ so the formula holds for $n=0$, so suppose that $n>0$. We prove by induction on $q$ a slightly stronger result: that

$$
f_{m}^{(n)}(a)=\frac{n !}{(n-q) !} f_{m+q}^{(n-q)}(a)
$$

holds for $m \geq 0, n>0$ and $0 \leq q \leq n$. Since $f_{m}^{(n)}(a)=$ $(n ! / n !) f_{m}^{(n)}(a)$, (2) holds for $q=0$. Suppose now that (2) holds for a given $q, 0 \leq q<n$. From the definition of $S_{f}$, we have $f_{m+q}=(x-a) f_{m+q+1}+f_{m+q}(a)$. And $n-q>0$, so, by Lemma 2 , $f_{m+q}^{(n-q)}(a)=(n-q) f_{m+q+1}^{(n-q-1)}(a)$. Thus,

$$
f_{m}^{(n)}(a)=\frac{n !}{(n-q) !} f_{m+q}^{(n-q)}(a)=\frac{n !}{(n-(q+1)) !} f_{m+(q+1)}^{(n-(q+1))}(a)
$$

so (2) holds for $q+1$.

Plugging in $m=0$ and $q=n$ in (2), we get

$$
f^{(n)}(a)=f_{0}^{(n)}(a)=n ! f_{n}(a)
$$

which is Taylor's formula.

Since Taylor's formula holds in $k$, the $P$-adic expansions for elements of $\mathcal{O}_{a}$ are exactly the Taylor series expansions for complex rational functions.

Let $f \in k(x)$ and suppose that $f \notin \mathcal{O}_{a}$. Then $\nu=-\nu_{a}(f)>0$. $\nu$ is called the order of the pole of $f$ at $x=a$. Let $f_{a}=(x-a)^{\nu} f$. Then, $\nu_{a}\left(f_{a}\right)=\nu+\nu_{a}(f)=0$, so $f_{a} \in \mathcal{O}_{a}$, so $f_{a}$ has a $P$-adic expansion at $x=a$ of the form $\sum_{n=0}^{\infty} b_{n}(x-a)^{n}$. We define the $P$-adic expansion of $f$ at $x=a$ to be the series

$$
\sum_{n=\nu_{a}(f)}^{\infty} a_{n}(x-a)^{n}=\frac{a_{-\nu}}{(x-a)^{\nu}}+\ldots+\frac{a_{-1}}{(x-a)}+a_{0}+a_{1}(x-a)+\cdots
$$

where $a_{n}=b_{n+\nu}$ for $n \geq \nu_{a}(f)$. Applying Taylor's formula to $f_{a}$, we find

$$
a_{n}=\frac{f_{a}^{\left(n-\nu_{a}(f)\right)}(a)}{\left(n-\nu_{a}(f)\right) !} \text { for } n \geq \nu_{a}(f) .
$$


The $P$-adic expansions for elements of $k(x) \backslash \mathcal{O}_{a}$ are exactly the Laurent series expansions for complex rational functions. The coefficient $a_{-1}$ is called the residue of $f$ at $x=a$. We extend this terminology to the other coefficients.

Definition 2 Let $f \in k(x)$ and $m>0$. The residue of order $m$ of $f$ at $x=a$ is the coefficient of $(x-a)^{-m}$ in the P-adic expansion of $f$ at $x=a$.

The residues of order 1 of $f$ are just called the residues of $f$ and correspond to the usual residues for complex functions.

\section{Rational computation of the residues}

We show in this section that we can compute the residues of $f$ of any order without factoring the denominator of $f$ or extending the coefficient field of $f$.

Theorem 1 Let $K$ be a field of characteristic $0, \bar{K}$ be an algebraic closure of $K$, and $x$ be transcendental over $K$. Then, for any $f \in K(x)$ and any integers $n>0$ and $0<m \leq n$, there exists a polynomial $B_{n m}(f) \in K(x)$ whose roots in $\bar{K}$ are exactly the residues of order $m$ of $f$ at all its poles of order $n$ in $\bar{K}$. Furthermore $B_{n m}(f)$ can be computed using only rational operations over $K$.

Proof. We give an algorithm that computes $B_{n m}(f)$. Let $f \in K(x)$ and write $f=A / D$ where $A, D \in K[x],(A, D)=(1)$, and $D$ is monic. Let $D=D_{1} D_{2}{ }^{2} \ldots D_{p}{ }^{p}$ be a squarefree factorisation of $D$. Let $n$ and $m$ be integers with $n>0$ and $0<m \leq n$. If $n>p$ or $D_{n} \in K$, then $f$ has no pole of order $n$ in $\bar{K}$, so $B_{n m}(f)=x$. Suppose now that $n \leq p$ and $\operatorname{deg}\left(D_{n}\right) \geq 1$. Let $t, v_{0}, v_{1}, \ldots, v_{n-m}$ be indeterminates over $K(x)$, and $u$ be a differential indeterminate over $K(x)$. Compute

$$
h=\frac{\left(f D_{n}{ }^{n} u^{-n}\right)^{(n-m)}}{(n-m) !} \in K(x)\langle u\rangle
$$

and write $h$ as

$$
h=\frac{P\left(x, u, u^{\prime}, u^{\prime \prime}, \ldots, u^{(n-m)}\right)}{Q\left(x, u, u^{\prime}, u^{\prime \prime}, \ldots, u^{(n-m)}\right)}
$$


where $P, Q \in K\left[x, v_{0}, v_{1}, \ldots, v_{n-m}\right]$. Compute then

$$
\tilde{P}=P\left(x, D_{n}^{\prime}, \frac{D_{n}^{\prime \prime}}{2}, \frac{D_{n}^{(3)}}{3}, \ldots, \frac{D_{n}^{(n-m+1)}}{n-m+1}\right) \in K[x]
$$

and

$$
\tilde{Q}=Q\left(x, D_{n}^{\prime}, \frac{D_{n}^{\prime \prime}}{2}, \frac{D_{n}{ }^{(3)}}{3}, \ldots, \frac{D_{n}{ }^{(n-m+1)}}{n-m+1}\right) \in K[x]
$$

We note that, since the only denominators involving $u$ in $h$ are powers of $u$, the $Q$ in formula (5) can be of the form $Q=u^{t} q$ for some integer $t>0$ and $q \in K[x]$. Hence $\tilde{Q}=\left(D_{n}^{\prime}\right)^{t} q$. Furthermore, $\nu_{\alpha}\left(f D_{n}^{n}\right) \geq 0$ for any $\alpha \in \bar{K}$ such that $D_{n}(\alpha)=0$, hence $\nu_{\alpha}(h) \gtrsim 0$ so $\left(q, D_{n}\right)=(1)$. Since $D_{n}$ is squarefree, this implies that $\left(\tilde{Q}, D_{n}\right)=(1)$. Let

$$
B_{n m}(f)=\operatorname{resultant}_{x}\left(\tilde{P}-t \tilde{Q}, D_{n}\right) \in K[t] .
$$

We now claim that the roots of $B_{n m}(f)$ in $\bar{K}$ are exactly the residues of order $m$ of $f$ at its poles of order $n$ in $\bar{K}$.

Let $\alpha_{1}, \ldots, \alpha_{q} \in \bar{K}$ be the distinct poles of $f$ of order $n$ in $\bar{K}$. Then $D_{n}=\prod_{i=1}^{q}\left(x-\alpha_{i}\right)$, so

$$
B_{n m}(f)=\gamma \prod_{i=1}^{q}\left(\tilde{P}\left(\alpha_{i}\right)-t \tilde{Q}\left(\alpha_{i}\right)\right)
$$

where $\gamma \in K \backslash\{0\}$, so the roots of $B_{n m}(f)$ in $\bar{K}$ are exactly $\tilde{P}\left(\alpha_{i}\right) / \tilde{Q}\left(\alpha_{i}\right)$ for $i=1 \ldots q\left(\tilde{Q}\left(\alpha_{i}\right) \neq 0\right.$ since $\left.\left(\tilde{Q}, D_{n}\right)=(1)\right)$. Let $\alpha$ be one of the $\alpha_{i}$ 's, and $D_{\alpha}=D_{n} /(x-\alpha) \in \bar{K}[x]$. Let also $g_{\alpha}=f D_{n}{ }^{n} D_{\alpha}{ }^{-n} \in \bar{K}(x)$, and $h_{\alpha}=g_{\alpha}{ }^{(n-m)} /((n-m) !) \in \bar{K}(x)$. We then have $h_{\alpha}=\bar{P} / \bar{Q}$ where

$$
\bar{P}=P\left(x, D_{\alpha}, D_{\alpha}^{\prime}, D_{\alpha}^{\prime \prime}, \ldots, D_{\alpha}^{(n-m)}\right) \quad \in \bar{K}[x]
$$

and

$$
\bar{Q}=Q\left(x, D_{\alpha}, D_{\alpha}^{\prime}, D_{\alpha}^{\prime \prime}, \ldots, D_{\alpha}{ }^{(n-m)}\right) \quad \in \bar{K}[x]
$$

and $P$ and $Q$ are given by (5). We have $D_{n}=(x-\alpha) D_{\alpha}$, so, by Lemma $2, D_{n}{ }^{(j)}(\alpha)=j D_{\alpha}{ }^{(j-1)}(\alpha)$ for any $j>0$, hence $\tilde{P}(\alpha)=$ $\bar{P}(\alpha)$ and $\tilde{Q}(\alpha)=\bar{Q}(\alpha)$. 
Thus, the roots of $B_{n m}(f)$ are exactly $h_{\alpha_{i}}\left(\alpha_{i}\right)$ for $i=1 \ldots q$. From formula (3), the residue of order $m$ of $f$ at $x=\alpha$ is

$$
a_{\alpha,-m}=\frac{f_{\alpha}{ }^{(n-m)}(\alpha)}{(n-m) !}
$$

where $f_{\alpha}=(x-\alpha)^{n} f$. But $(x-\alpha) D_{\alpha}=D_{n}$, so

$$
f_{\alpha}=\frac{D_{n}^{n}}{D_{\alpha}{ }^{n}} f=g_{\alpha}
$$

so $h_{\alpha}=f_{\alpha}{ }^{(n-m)} /(n-m)$ !, so $h_{\alpha}(\alpha)=a_{\alpha,-m}$. Hence, the roots of $B_{n m}(f)$ in $\bar{K}$ are exactly the residues of order $m$ of $f$ at $\alpha_{1}, \ldots, \alpha_{q}$.

For efficiency, and since dividing $\tilde{P}$ and $\tilde{Q}$ by their gcd does not change the roots of $B_{n m}(f)$, it is better to replace formula (6) by

$$
B_{n m}(f)=\text { resultant }_{x}\left(\frac{\tilde{P}}{G}-t \frac{\tilde{Q}}{G}, D_{n}\right)
$$

where $G=\operatorname{gcd}(\tilde{P}, \tilde{Q}) \in K[x]$.

As a consequence, we can compute a polynomial whose roots are exactly all the (usual) residues of $f$ at all its poles.

Corollary 1 (Residues) The residues of $f$ at all its poles in $\bar{K}$ are exactly all the roots in $\bar{K}$ of $R(f)=\Pi_{n=1}^{p} B_{n 1}(f) \in K[t]$ where the $B_{n 1}$ 's are given by (6).

In addition, it is often useful to compute quantities involving both the residues and the poles at which they appear. It is clear from the proof of Theorem 1 that multiple roots of $B_{n m}(f)$ correspond to residues of order $m$ appearing at several poles of order $n$ of $f$. The following Theorem, which generalizes the result of [4], shows that one can separate the poles of order $n$ into subsets having the same residue of order $m$ without extending $K$.

Theorem 2 Let $K$ be a field of characteristic $0, \bar{K}$ be an algebraic closure of $K$, and $x$ be transcendental over $K$. Let $f \in K(x)$ and $n>0$ and $0<m \leq n$ be integers. Let $1 \leq i \leq \operatorname{deg}\left(B_{n m}(f)\right)$ and $G_{n m i}(f) \in K[x ; t]$ be the remainder of degree $i$ in $x$ appearing in the computation of $B_{n m}(f)$ by formula (6) via the subresultant algorithm. Then, for any root $\alpha$ of multiplicity $i$ of $B_{n m}(f)$ in $\bar{K}$, $\operatorname{deg}\left(G_{n m i}(f)(x, \alpha)\right)=i$, and its roots in $\bar{K}$ are exactly the poles of order $n$ of $f$ in $\bar{K}$ where the residue of order $m$ is equal to $\alpha$. 
Proof. The proof follows exactly the one of [4]. Let the notation be as in the proof of Theorem 1. Let $\alpha \in \bar{K}$ be a root of multiplicity $i$ of $B_{n m}(f)$. By Proposition 3 of [4], we have

$$
G_{n m i}(f)(x, \alpha)=\operatorname{gcd}\left(\tilde{P}-\alpha \tilde{Q}, D_{n}\right) .
$$

The roots of the above gcd are exactly the roots $a \in \bar{K}$ of $D_{n}$ such that $\tilde{P}(a)-\alpha \tilde{Q}(a)=0$. As in the proof of Theorem 1. $\tilde{P}(a) / \tilde{Q}(a)$ is the residue of order $m$ at $x=a$. Furthermore, the roots of $D_{n}$ are exactly the poles of order $n$ of $f$. Hence, the roots of $G_{n m i}(x, \alpha)$ in $\bar{K}$ are exactly the poles of order $n$ of $f$ in $\bar{K}$ where the residue of order $m$ is equal to $\alpha$.

\section{Special cases}

For any specific values of $n$ and $m$, we can compute a closed-form formula for $B_{n m}(f)$ in terms of its numerator and denominator, by applying the algorithm of the previous section with symbolic $A$ and $D_{n}$. We describe in this section the cases for $m=n$ which corresponds to the leading coefficient of the Laurent series expansions of $f$, and for $m=n-1$.

Let $K$ be a field of characteristic $0, \bar{K}$ be an algebraic closure of $K, x$ and $t$ be indeterminates over $K$, and $f \in K(x)$. For all of this section we write $f=A / D$ where $A, D \in K[x],(A, D)=(1)$ and $D$ is monic. We also let $D=D_{1} D_{2}{ }^{2} \ldots D_{p}{ }^{p}$ be a squarefree factorisation of $D$. Finally, we write $D_{n}^{*}=D / D_{n}{ }^{n}$ for $n=1 \ldots p$. Corollary 2 (Leading Coefficients) Let $n \in \mathbf{Z}, 1 \leq n \leq p$. Then, the leading coefficients of the Laurent series of $f$ at all its poles of order $n$ in $\bar{K}$ are exactly all the roots in $\bar{K}$ of

$$
L_{n}(f)=\text { resultant }_{x}\left(A-t D_{n}^{*} D_{n}^{\prime n}, D_{n}\right) \in K[t] .
$$

Proof. Plugging in $m=n$ in the algorithm of the previous section, (4) becomes $h=g=f D_{n}{ }^{n} u^{-n}=A /\left(D_{n}^{*} u^{n}\right)$, so $\tilde{P}=A$ and $\tilde{Q}=D_{n}^{*} D_{n}^{\prime n}$. Hence $L_{n}(f)=B_{n n}(f)$, so its roots are the leading coefficients of the Laurent series of $f$ at all its poles of order $n$ in $\bar{K}$.

We note that if $D$ is squarefree, then the roots of $L_{1}(f)$ are exactly all the residues of $f$ in $\bar{K}$, and that

$$
L_{1}(f)=\text { resultant }_{x}\left(A-t D^{\prime}, D\right)
$$


in that case, and this is precisely the Trager-Rothstein resultant used in symbolic integration algorithms $[6,7]$.

Corollary 3 Let $n \in Z, 2 \leq n \leq p$. Then, the residues of order $n-1$ of $f$ at all its poles of order $n$ in $\bar{K}$ are exactly all the roots in $\bar{K}$ of

$M_{n}(f)=$ resultant $_{x}\left(A^{\prime} D_{n}^{*} D_{n}^{\prime}-A D_{n}^{* \prime} D_{n}^{\prime}-\frac{n}{2} A D_{n}^{*} D_{n}^{\prime \prime}-t D_{n}^{* 2} D_{n}^{\prime n+1}, D_{n}\right)$.

Proof. Plugging in $m=n-1$ in the algorithm of the previous section, (4) becomes

$$
h=\left(\frac{A}{D_{n}^{*} u^{n}}\right)^{\prime}=\frac{A^{\prime} D_{n}^{*} u-A D_{n}^{* \prime} u-n A D_{n}^{*} u^{\prime}}{D_{n}^{* 2} u^{n+1}}
$$

so the above formula for $M_{n}(f)$ is just formula (6) for $m=n-1$, so $M_{n}(f)=B_{n, n-1}(f)$.

It is clear from the above corollary that a closed form formula for $B_{n m}(f)$ can be derived in terms of $n$ for any particular values of $n-m$. The size of these formulas grows however with a rate similar to the size of $\left(u^{n}\right)^{(q)}$ as $q$ goes from 0 to $n$.

\section{Computation of the principal parts}

Let $K$ be a field of characteristic $0, x$ an indeterminate over $K$, $\bar{K}$ an algebraic closure of $K$, and $f \in k(x)$. From Theorem 1, the field generated by the coefficients of the principal parts of the Laurent series expansions of $f$ at all its poles in $\bar{K}$ is the common splitting field of all the $B_{i j}^{\prime} s$ over $K$. Furthermore, as a consequence of Theorem 2, we can compute all those principal parts while working only in the splitting fields of the $B_{i j}$ 's as follows: write $f=A / D$ where $A, D \in K[x], D$ is monic and $(A, D)=(1)$. Let $D=D_{1} D_{2}^{2} \ldots D_{n}^{n}$ be a squarefree factorization of $D$.

Compute then $B_{11}(f)$ and let $\left(\alpha_{i j}\right)_{i, j}$ be all its distinct roots in $\bar{K}$, with the notation such that each $\alpha_{i j}$ is a zero of multiplicity $i$ of $B_{11}(f)$. This yields the following factorization of $D_{1}$ :

$$
D_{1}=\prod_{i} \prod_{j} G_{11 i}\left(x, \alpha_{i j}\right)
$$


where for each $i$ and $j$, the principal parts of $f$ at a pole $\beta$ such that $G_{11 i}\left(\beta, \alpha_{i j}\right)=0$ are

$$
f=\frac{\alpha_{i j}}{x-\beta}+\ldots
$$

Repeating the same procedure with $B_{22}(f)$ yields a factorisation of $D_{2}$ and the leading terms of the Laurent series expansions of $f$ at its double poles. Splitting then $B_{21}(f)$ refines this factorisation of $D_{2}$ and yields the complete principal parts of $f$ at its double poles. After doing this for $B_{i j}(f)$ for $1 \leq j \leq i \leq n$, we get a factorisation of $D$ into factors having the same principal parts and the principal parts of $f$ at all those factors. All the computations are done in the splitting fields of the $B_{i j}^{\prime} s$ over $K$.

Example: Consider

$$
f=\frac{2 x^{7}-7 x^{5}+26 x^{3}+8 x}{x^{8}-5 x^{6}+6 x^{4}+4 x^{2}-8} \in \mathbf{Q}(x) .
$$

The squarefree factorisation of the denominator of $f$ returns

$$
D=D_{1} D_{3}^{3}=\left(x^{2}+1\right)\left(x^{2}-2\right)^{3}
$$

so $f$ has poles at $x= \pm \sqrt{-1}$ and $x= \pm \sqrt{2}$.

For $n=m=1$, we have $D_{1}=x^{2}+1$, so (4) becomes

$$
h=\frac{f D_{1}}{u}=\frac{2 x^{7}-7 x^{5}+26 x^{3}+8 x}{\left(x^{6}-6 x^{4}+12 x^{2}-8\right) u} .
$$

$D_{1}^{\prime}=2 x$, so we get $\tilde{P}=2 x^{7}-7 x^{5}+26 x^{3}+8 x, \tilde{Q}=2 x^{7}-12 x^{5}+$ $24 x^{3}-16 x$, and $G=x$. Hence,

$$
B_{11}(f)=\text { resultant }_{x}\left(\frac{\tilde{P}}{G}-t \frac{\tilde{Q}}{G}, x^{2}+1\right)=729(2 t-1)^{2} .
$$

$B_{11}$ has a double zero $t=1 / 2$, and the remainder of degree 2 in $x$ in the subresultant algorithm is $G_{112}(f)(x, t)=x^{2}+1$, so $G_{112}(f)(x, 1 / 2)=x^{2}+1$ so the principal parts of the Laurent series of $f$ at $\alpha^{2}+1=0$ are

$$
f=\frac{1 / 2}{(x-\alpha)}+\ldots
$$


For $n=3$, we have $D_{3}=x^{2}-2$, so for $m=1$, (4) becomes

$$
h=\frac{f D_{3}^{3}}{u^{3}}=\frac{2 x^{7}-7 x^{5}+26 x^{3}+8 x}{\left(x^{2}+1\right) u^{3}} .
$$

$D_{3}^{\prime}=2 x$, so we get $\tilde{P}=2 x^{7}-7 x^{5}+26 x^{3}+8 x, \tilde{Q}=8 x^{5}+8 x^{3}$, and $G=x$. Hence,

$$
B_{33}(f)=\text { resultant }_{x}\left(\frac{\tilde{P}}{G}-t \frac{\tilde{Q}}{G}, x^{2}-2\right)=2304(t-1)^{2} .
$$

$B_{33}$ has a double zero $t=1$, and the remainder of degree 2 in $x$ in the subresultant algorithm is $G_{332}(f)(x, t)=x^{2}-2$.

For $m=2$, we differentiate (7) once, obtaining $h=\frac{10 x^{8}-7 x^{6}-9 x^{4}+70 x^{2}+8}{\left(x^{4}+2 x^{2}+1\right) u^{2}}-\frac{\left(6 x^{9}-15 x^{7}+57 x^{5}+102 x^{3}+24 x\right) u^{\prime}}{\left(x^{4}+2 x^{2}+1\right) u^{3}}$.

$D_{3}^{\prime \prime}=2$ so we get $\tilde{P}=14 x^{9}+x^{7}-75 x^{5}+38 x^{3}-8 x, \tilde{Q}=16 x^{8}+$ $32 x^{6}+16 x^{4}$, and $G=x$. Hence,

$$
B_{32}=\text { resultant }_{x}\left(\frac{\tilde{P}}{G}-t \frac{\tilde{Q}}{G}, x^{2}-2\right)=-165888 t^{2} .
$$

$B_{32}$ has a double zero $t=0$, and the remainder of degree 2 in $x$ in the subresultant algorithm is $G_{322}(f)(x, t)=x^{2}-2$.

For $m=1$, we differentiate (7) twice and divide by 2!, obtaining a formula for $h$ in terms of $x, u, u^{\prime}$ and $u^{\prime \prime}$. Replacing $u$ by $2 x$, $u^{\prime}$ by 1 and $u^{\prime \prime}$ by 0 , we get $\tilde{P}=2 x^{6}+6 x^{4}+6 x^{2}-25, \tilde{Q}=$ $2 x^{6}+6 x^{4}+6 x^{2}+2$, and $G=1$. Hence,

$$
B_{31}=\text { resultant }_{x}\left(\frac{\tilde{P}}{G}-t \frac{\tilde{Q}}{G}, x^{2}-2\right)=729(2 t-1)^{2} .
$$

$B_{31}$ has a double zero $t=1 / 2$, and the remainder of degree 2 in $x$ in the subresultant algorithm is $G_{312}(f)(x, t)=x^{2}-2$.

We find that

$$
G_{332}(f)(x, 1)=G_{322}(f)(x, 0)=G_{312}(f)(x, 1 / 2)=x^{2}-2
$$

so the principal parts of the Laurent series of $f$ at $\beta^{2}-2=0$ are

$$
f=\frac{1}{(x-\beta)^{3}}+\frac{1 / 2}{(x-\beta)}+\ldots
$$


Hence, the residues of $f$ are exactly $1 / 2$ at all its poles, and its leading coefficients are 1 at both its triple poles. Furthermore, its residues of order 2 are 0 at these poles. In this particular case, all the coefficients of the principal parts of $f$ are rational numbers, so we could reconstruct all of them computing only in $\mathbf{Q}(x)$.

The algorithm of this section has been implemented in the Maple computer algebra system by B. Salvy (INRIA Roquencourt, France). The following is an interactive session computing the Laurent series for the above example:

$>\mathrm{f}:=\left(2 * \mathrm{x}^{\wedge} 7-7 * \mathrm{x}^{\wedge} 5+26 * \mathrm{x}^{\wedge} 3+8 * \mathrm{x}\right) /\left(\mathrm{x}^{\wedge} 8-5 * \mathrm{x}^{\wedge} 6+6 * \mathrm{x}^{\wedge} 4+4 * \mathrm{x}^{\wedge} 2-8\right)$;

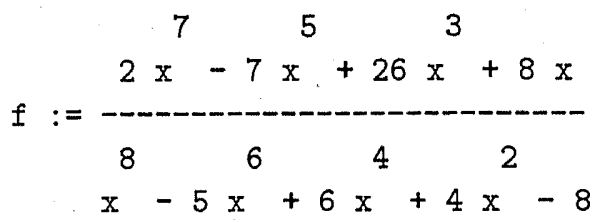

$>$ fullparfrac $(f, x)$;
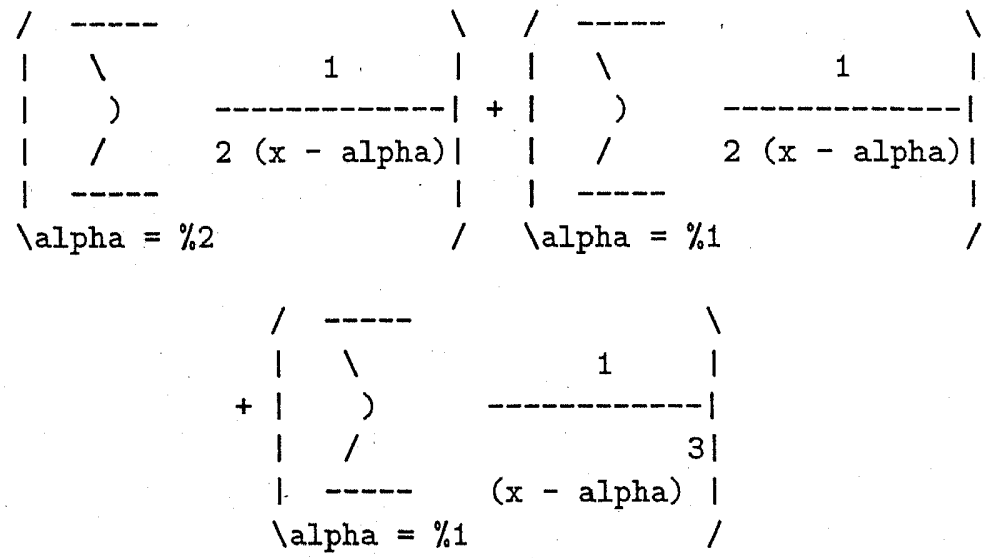
$\% 1:=$
$\operatorname{RootOf}\left(\_Z-2\right)$
2
$\% 2:=$
$\operatorname{Root0f}(-Z+1)$ 


\section{Kovacic's necessary conditions}

Kovacic's algorithm [1] computes a closed form solution (if it exists) of an equation of the form $y^{\prime \prime}=r y$ where $r \in \mathbf{C}(x)$ is given and ${ }^{\prime}=d / d x$. The algorithm has 3 cases, corresponding to 3 possible types of closed-form solutions, the $4^{\text {th }}$ case being that the equation has no closed-form (Liouvillian) solution. In order for the algorithm to be more effective and to avoid going through all the cases, Kovacic proves that the following conditions on $r$ are necessary for each of the 3 cases respectively:

Theorem 3 (Kovacic). The following conditions are necessary for the respective cases to hold.

Case 1. Every pole of $r$ must have order 1 or even order. The order of $r$ at $\infty$ must be even or greater than 2.

Case 2. $r$ must have at least one pole that either has order 2 or odd order greater than 2.

Case 3. The order of a pole of $r$ cannot exceed 2 and the order of $r$ at $\infty$ must be at least 2 . If the partial fraction expansion of $r$ is

$$
r \doteq \sum_{i} \frac{\alpha_{i}}{\left(x-c_{i}\right)^{2}}+\sum_{j} \frac{\beta_{j}}{x-d_{j}}
$$

then $\sqrt{1+4 \alpha_{i}} \in \mathbf{Q}$ for each $i, \Sigma_{j} \beta_{j}=0$ and $\sqrt{1+4 \gamma} \in \mathbf{Q}$ where

$$
\gamma=\sum_{i} \alpha_{i}+\sum_{j} \beta_{j} d_{j}
$$

In general, $r \in K(x)$ where $K$ is a finitely generated extension of the rational number field. As a consequence of Theorem 1 , we can check alternative necessary conditions that require computations in $K$ extended by the $\beta_{j}$ 's only. For any polynomial $C=a_{n} x^{n}+$ $a_{n-1} x^{n-1}+\ldots+a_{0} \in K[x]$ with $a_{n} \neq 0$ and $n>0$, we define $\operatorname{trace}(C)=-a_{n-1} / a_{n}$. It is a well known result (e.g. [2]) that $\operatorname{trace}(C)$ is equal to the sum of the roots of $C$ in $\bar{K}$.

Theorem 4 Let $r \in K(x)$ and write $r=A / D$ where $A, D \in$ $K[x],(A, D)=(1)$ and $D$ is monic. Let $D=D_{1} D_{2}{ }^{2} \ldots D_{n}{ }^{n}$ be a 
squarefree factorisation of $D$. Then, the following conditions are necessary for the respective cases of Kovacic's algorithm to hold.

1. $\operatorname{deg}\left(D_{2 i+1}\right)=0$ for any $i \geq 1$, and $\operatorname{deg}(D)-\operatorname{deg}(A)$ is even or greater than 2.

2. $\operatorname{deg}\left(D_{2}\right)>0$ or $\operatorname{deg}\left(D_{2 i+1}\right)>0$ for some $i \geq 1$.

3. $n \leq 2$ and $\operatorname{deg}(D)-\operatorname{deg}(A) \geq 2$. Let

$$
P=\text { resultant }_{x}\left(A-t D_{1} D_{2}^{\prime 2}, D_{2}\right),
$$

$Q=\left\{\begin{array}{ccc}1 & \text { if } & \operatorname{deg}\left(D_{2}\right)=0 \\ \text { resultant }_{x}\left(\left(A D_{1} D_{2}^{\prime}\right)^{\prime}-t D_{1}{ }^{2} D_{2}^{\prime 3}, D_{2}\right) & \text { if } & \operatorname{deg}\left(D_{2}\right)>0\end{array}\right.$

$Q=Q_{1} Q_{2}{ }^{2} \ldots Q_{m}{ }^{m}$ be a squarefree factorisation of $Q, G_{i} \in$ $K[x, t]$ for $1 \leq i \leq m$ be the remainder of degree $i$ in $x$ in the computation of $Q$ via the subresultant algorithm,

$$
R=\text { resultant }_{x}\left(A-t D_{2}^{2} D_{1}^{\prime}, D_{1}\right),
$$

$R=R_{1} R_{2}{ }^{2} \ldots R_{p}{ }^{p}$ be a squarefree factorisation of $R$, and $H_{j} \in K[x, t]$ for $1 \leq j \leq p$ be the remainder of degree $j$ in $x$ in the computation of $R$ via the subresultant algorithm.

Then, $\operatorname{trace}(Q R)=0, \operatorname{prim}_{x}(P) \in \mathrm{Q}[x]$ and it factors linearly over $\mathbf{Q}, \sqrt{1+4 \alpha} \in \mathbf{Q}$ for each root $\alpha$ of $P$, and $\sqrt{1+4 \gamma} \in \mathbf{Q}$ where

$$
\begin{aligned}
\gamma=\operatorname{trace}(P) & +\sum_{i=1}^{m} \sum_{Q_{i}(\beta)=0} \beta \operatorname{trace}\left(G_{i}(x, \beta)\right) \\
& +\sum_{j=1}^{p} \sum_{R_{j}(\beta)=0} \beta \operatorname{trace}\left(H_{j}(x, \beta)\right) .
\end{aligned}
$$

Proof. The conditions for cases 1 and 2 are direct rewriting of Kovacic's corresponding conditions in terms of $A$ and the $D_{i}$ 's, so consider the condition for case 3 . That $n \leq 2$ is equivalent to saying that the order of a pole of $r$ cannot exceed 2 , and that $\operatorname{deg}(D)-\operatorname{deg}(A) \geq 2$ is equivalent to the order of $r$ at $\infty$ being at least 2 .

Since $n \leq 2, D_{2}^{*}=D_{1}$, so, by Corollary 2 , the roots of $P$ are exactly the $\alpha_{i}$ 's of (8). Hence, $\sqrt{1+4 \alpha_{i}} \in \mathbf{Q}$ for each $i$ is equivalent 
to saying that $\sqrt{1+4 \alpha} \in \mathbf{Q}$ for each root $\alpha$ of $P$. This implies that each root of $P$ is in $\mathbf{Q}$, hence that $\operatorname{prim}_{x}(P)$ factors linearly over $\mathbf{Q}$ and is in $\mathbf{Q}[x]$ since its coefficients are symmetric functions of its roots.

We also have $D_{1}^{*}=D_{2}{ }^{2}$, so, by Corollary 2 , the roots of $R$ are exactly the $\beta_{j}$ 's of (8) at the zeroes of $D_{1}$, and, by Corollary 3 , the roots of $Q$ are exactly the $\beta_{j}$ 's of (8) at the zeroes of $D_{2}$. Since $\left(D_{1}, D_{2}\right)=(1)$, we get $\Sigma_{j} \beta_{j}=\operatorname{trace}(Q R)$, so $\operatorname{trace}(Q R)=0$ is equivalent to $\Sigma_{j} \beta_{j}=0$.

Since the roots of $P$ are the $\alpha_{i}^{\prime}$ 's, we have $\sum_{i} \alpha_{i}=\operatorname{trace}(P)$. Now let $\beta$ be a root of $Q_{i}$ in $\bar{K}$. By Theorem 2, the roots of $G_{i}(x, \beta)$ are exactly those $d_{j}$ 's such that $D_{2}\left(d_{j}\right)=0$ and $\beta_{j}=\beta$. Since $Q_{i}$ is squarefree and $\left(Q_{i}, Q_{k}\right)=(1)$ for $i \neq k$, we get

$$
\sum_{i=1}^{m} \sum_{Q_{i}(\beta)=0} \beta \operatorname{trace}\left(G_{i}(x, \beta)\right)=\sum_{D_{2}\left(d_{j}\right)=0} \beta_{j} d_{j} .
$$

By a similar argument, we get

$$
\sum_{j=1}^{p} \sum_{R_{j}(\beta)=0} \beta \operatorname{trace}\left(H_{j}(x, \beta)\right)=\sum_{D_{1}\left(d_{j}\right)=0} \beta_{j} d_{j} .
$$

Hence,

$$
\begin{aligned}
\sum_{i} \alpha_{i}+\sum_{j} \beta_{j} d_{j}=\operatorname{trace}(P) & +\sum_{i=1}^{m} \sum_{Q_{i}(\beta)=0} \beta \operatorname{trace}\left(G_{i}(x, \beta)\right) \\
& +\sum_{j=1}^{p} \sum_{R_{j}(\beta)=0} \beta \operatorname{trace}\left(H_{j}(x, \beta)\right) .
\end{aligned}
$$

\section{References}

[1] Kovacic J.J.: An Algorithm for Solving Second Order Linear Homogeneous Differential Equations. Journal of Symbolic Computation, 2, 3-43 (1986)

[2] Lang, S.: Algebra (Ch. V). Reading, Massachusetts: AddisonWesley 1971

[3] Lang, S.: Introduction to Algebraic and Abelian Functions (Ch. I). Reading, Massachusetts: Addison-Wesley 1972 
[4] Lazard, D., Rioboo, R.: Integration of Rational Functions: Rational Computation of the Logarithmic Part. Journal of Symbolic Computation, 9, 113-116 (1990)

[5] Marsden, J.E.: Basic Complex Analysis (Ch. 4). San Francisco, California: W.H.Freeman and Co. 1973

[6] Rothstein, M.: A New Algorithm for the Integration of Exponential and Logarithmic Functions. In: Proceedings 1977 MACSYMA Users Conference, NASA Pub. CP-2012, 263-274 (1977)

[7] Trager, B.: Algebraic Factoring and Rational Function Integration. In: Proceedings SYMSAC '76, 219-226 (1976) 